\title{
A Comparative Analysis of Asymmetrical U-Slot and Substrate Integrated Waveguide Fed Microstrip Patch Antenna
}

\author{
Neha Pathak \\ Lakshmi Narain College of Technology Excellence \\ Bhopal, India
}

\begin{abstract}
This paper shows a comparative study of Multi U-shaped slot antenna with the substrate integrated waveguide(SIW) fed patch antenna. By cutting four asymmetrical U-slots, we can make U-shaped patch antenna. Advantages of this structure are the simple feed, single layer structure, simple structure of antenna, one more degree of freedom can be achieved by the asymmetry of U-slot arm. The design guideline for the proposed antenna is given and the acceptability of the design is verified by other scenarios. When we create two different frequency bands with distinct polarization, two longitudinal and transverse slots on broad wall of SIW of other antenna is responsible for it. For reducing the cross polarization level of antenna, frequency selective surface (FSS) is placed on top of the microstip patches. For getting the wider impedance bandwidth, we do close the resonance frequencies of the patch and slot to each other. Efficiency of the antenna can be increases by SIW feeding network.
\end{abstract}

\section{Keywords}

Linear polarization, single-layer, single-patch, U-slot, fourband, frequency selective surfaces, patch antennas, substrate integrated waveguide.

\section{INTRODUCTION}

Since many years ago these multiband communication, multifunction application and miniaturization has proposed. The most advantageous of multi band system is that when interference in one band system, the other can still work. Complementary function can be performed at the same time whenever we deal with uplink and downlink data in satellite communication. Multiband antenna is helpful in it. From past ten years by having multilayer, cutting slots in the patch, we can integrate multiband performance into one antenna[3].By cutting five opened ended slots, a five band linear polarization antenna was getted in the printed substrate[4].Between second and third bands, peak refection coefficient was only $8 \mathrm{db}$. In [5] various properties has presented like a compact multiband antenna, rearranging a planar monopole, a parasitic element, a novel T shaped element. $2.5 \mathrm{dbi}$ and $1.5 \mathrm{dbi}$ are the peak gains of antenna in lower and higher bands. By introducing of new dual layer slotted patch antenna, dual band performance has realized and it is applied in downlink and uplink satellite communication [6].

Between [1] and [2] mobile phone Bluetooth, GPS navigation, WLAN and Wi-Max, U slot patch antennas have been introduced. Mainly these designs can give only one and two bands. By increasing number of information services, single frequency antennas no longer meet the current market demands. What is exactly required for modern communication are a multiband multifunction, simple structure and low cost antenna. Various solutions are having including microstrip

\author{
Abhinav Bhargava \\ Lakshmi Narain College of Technology Excellence \\ Bhopal, India
}

\begin{abstract}
feed structure, single layer multi slot multiband patch antenna, multi layer stacked patch structure. But among all these we opt for single layer multi slot multiband patch antenna. And single layer multi slot multiband patch antenna has following advantages like high gain, band isolation, simple structure and easy to integrate on planar and non planar surfaces. In WLAN and Wi-max system a single layer single band coaxial probe $U$ slot antenna has used. The most advantageous of the $\mathrm{U}$ slot structure is by changing the length of the $U$ slot arm and the wideband performance, linear polarization and circular polarization radiation can be achieved. By cutting four asymmetrical U slots in the patch, we can made low cost, four band linear polarization $U$ slot patch antenna. Frequency bands worked for Wi-Max (3.3-3.8 GHz) and WLAN (5.150$5.250,5.250-5.350,5.725-5.825 \mathrm{GHz})$.Three of these u slots were used to introduce band notches at specific frequency points.
\end{abstract}

The ability of a system to receive and transmit signals with different polarizations is said to be POLARIZATION diversity. During in recent years dual polarized antennas has paid much attention by providing this capability for systems [7]. For getting this desired feature of antenna, several studies have used in microstrip antennas [8],[9].Developing different polarization in a same or different frequency band or one port to create two different bands, each having a different polarization, it can be achieved. By exciting two orthogonal modes, dual linear polarization is able to complete for microstrip patch antennas. A dual orthogonal linear polarizations can be generated by a new dual hybrid feed structure [10].A single feed dual polarized antenna is in [11].By adding a stub to the end of the feedline which stimulates two orthogonal modes of radiating patch.

\section{U- SLOTS ANTENNA DESIGN}

Four band linear polarization asymmetrical $\mathrm{U}$ slot patch antenna's geometry is shown in figure 1.In ground plane under the patch, a square copper plate was used along its edge having length $\mathrm{L}=50 \mathrm{~mm}$. Air is used as substrate and coaxial probe is connected directly to the patch. Inner and outer diameters of SMA connector were $0.6 \mathrm{~mm}$ and $2.0 \mathrm{~mm}$. HFSS is a commercial electromagnetic software was used in simulation).The final optimized parameters of the antenna are listed in Table 1. 
TABLE 1. Optimized dimensions of the antenna (unit: mm) central

\begin{tabular}{cccc}
\hline Parameter & Value & Parameter & Value \\
\hline $\mathbf{l}_{\mathbf{1}}$ & 16 & $\mathrm{w}_{2}$ & 18 \\
$\mathbf{l}_{\mathbf{2}}$ & 15 & $\mathrm{w}_{3}$ & 22.5 \\
$\mathbf{l}_{\mathbf{3}}$ & 9.5 & $\mathrm{w}_{4}$ & 26 \\
$\mathbf{l}_{\mathbf{4}}$ & 10 & $\mathrm{~h}_{1}$ & 7 \\
$\mathbf{l}_{\mathbf{5}}$ & 4.5 & $\mathrm{~h}_{2}$ & 5 \\
$\mathbf{l}_{\mathbf{6}}$ & 2 & $\mathrm{~h}_{3}$ & 3 \\
$\mathbf{l}_{\mathbf{7}}$ & 10.8 & $\mathrm{~h}_{4}$ & 1 \\
$\mathbf{l}_{\mathbf{8}}$ & 10 & $\mathrm{~W}$ & 38 \\
$\mathbf{m}_{\mathbf{1}}$ & 1.6 & $\mathrm{l}$ & 27.5 \\
$\mathbf{m}_{\mathbf{2}}$ & 1 & $\mathrm{~d}$ & 15.5 \\
$\mathbf{m}_{\mathbf{3}}$ & 1 & $\mathrm{~h}$ & 5 \\
$\mathbf{m}_{\mathbf{4}}$ & 1 & $\mathrm{~W}_{\mathrm{g}}$ & 50 \\
$\mathbf{m}_{\mathbf{5}}$ & 14.2 & $\mathrm{~L}_{\mathrm{g}}$ & 50 \\
\hline
\end{tabular}

The plan was that firstly design a $U$ slot patch antenna with broadband and then secondly to produce a notch at central frequency, for which total band can be divided into two sub bands. After that third and fourth $U$ slots are used to produce two notches at central frequencies of the two sub bands for which sub bands can be divided into four bands.

The central frequencies of the four bands required are $f_{1}=$ $3.30 \mathrm{GHz}, f_{2}=3.70 \mathrm{GHz}, f_{1}=5.20 \mathrm{GHz}$ and $f_{4}=5.80 \mathrm{GHz}$. Between two adjacent frequencies, notch frequencies were expected to be located at the centre. They were $3.5 \mathrm{GHz}, 4.45$ $\mathrm{GHz}$ and $5.5 \mathrm{GHz}$.

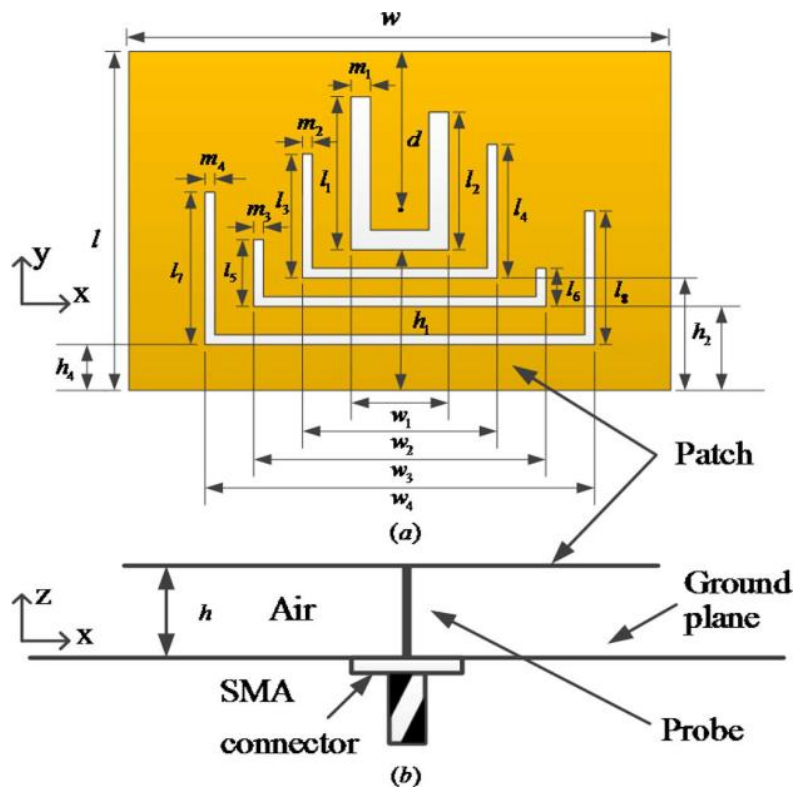

Fig 1: Geometry of the four-band patch antenna (a) Patch with four U-slots, (b) cross-sectional view [6].

$$
\begin{aligned}
& w=\frac{C}{2 f_{r}} \sqrt{\frac{2}{\varepsilon_{r}+1}} \\
& l=\frac{c}{2 f_{r \sqrt{\varepsilon_{r}}}}-2 \Delta l
\end{aligned}
$$

\section{ANTENNA DESIGN WITH (SIW)}

The configuration of the antenna is illustrated in Fig. 1, and the fabricated antenna is shown in fig. 2. It consists of three major parts. Feeding the patches by having two longitudinal and transverse slots etched on it, the SIW forms the lower portion. For lowering the cross polarization level of the antenna, top layer (frequency selective surface)is used and the middle part has two patches as radiating elements.SIW is implemented on Rogers 4003 with ,and thickness of $1.32 \mathrm{~mm}$. For blocking the slant pattern for frequency range, it will be shorted by vias and vias are having the distance of $1.5 \mathrm{~mm}$ in side walls and $1.6 \mathrm{~mm}$ in shorted walls and diameter is 0.8 $\mathrm{mm}$. Experimental equation given in [12].Conventional WR90 rectangular waveguide is having same behavior as SIW. Quarter wavelength should be away from SIW'S end, half should be transverse and longitudinal slots, for the effect of SIW's short wall impedance to be overlooked on the slots impedance .As shown

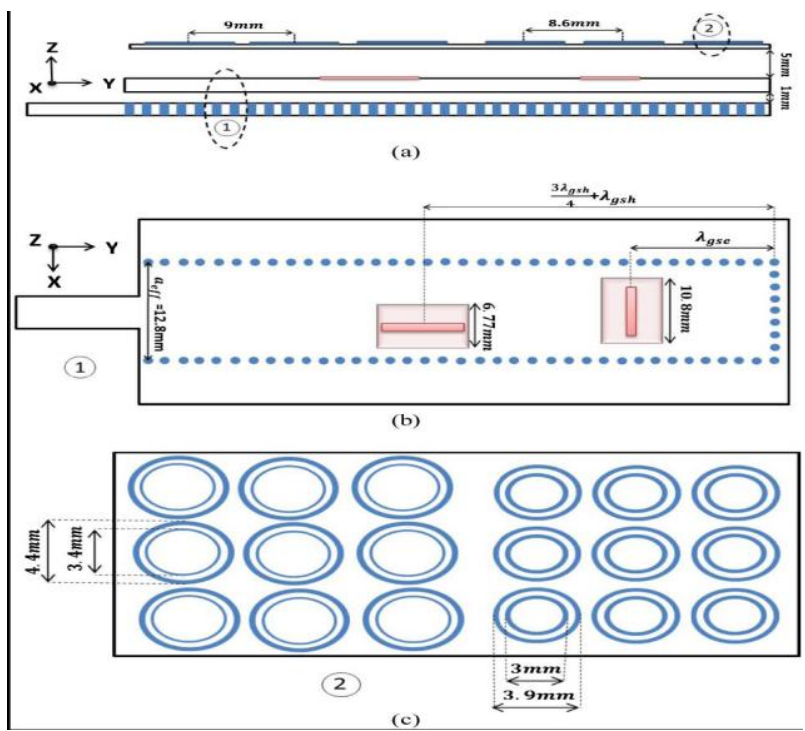

Fig 2: (a) Cross-sectional view (b) the position of the patches on slots (the bottom and middle layer), and (c) FSS (the top layer) of the antenna [7].

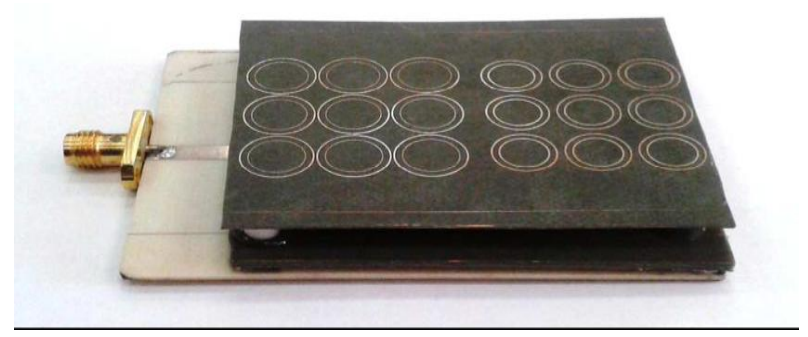

Fig 3: Prototype of the fabricated antenna in Fig 1(b), they have the required distance [7]

As shown in fig(b)required distance SIW's end to be locate at the standing wave peaks and then radiates well. Mainly important is that to meet up slot's distance requirements and at the end of SIW and should obey the equation of wavelength of two slots in their resonance frequency 


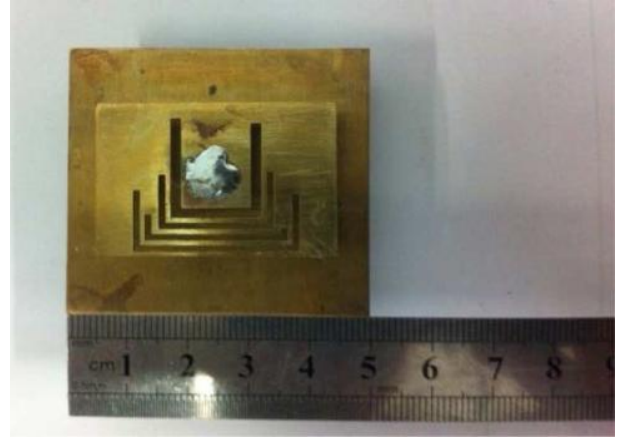

Fig 4: Photograph of the four-band antenna prototype.

$$
\frac{(2 n-1) \lambda_{g s h}}{4}=\frac{m \lambda_{g s e}}{2}
$$

Where $\lambda_{g s h}$ and $\lambda_{g s e}$ denotes wavelength of shunt and series patch loaded slots and they operates in different frequencies. resonance frequencies of loaded slots have values $\eta, \eta$ repeated periodically, to have distinct resonance frequencies in $\mathrm{X}$ band, two slots with their loaded patch are designed.

\section{SIMULATED AND MEASURED RESULTS}

This is for demonstrating the performance of the proposed antenna; simulation and measurement were agreed. Parametric studies were carried out to investigate the relationship between U-slot lengths and the notch-band frequencies. To investigate the relationship between $U$ slot lengths and the notch band frequencies, parametric studies were used. Simulated and measured reflection of proposed antenna shows in fig 4 . The measured impedance bandwidths with $-10 \mathrm{~dB} \mathrm{~dB}$ reflection coefficients of the four bands were $2.1 \%, 3.3 \%, 7.1 \%$ and $5.0 \%$, and those of the simulated ones $2.0 \%, 3.3 \%, 4.5 \%$ and $5.0 \%$. The measured center frequencies corresponding to WiMax and WLAN bands were $3.35 \mathrm{GHz}$, $3.70 \mathrm{GHz}, 5.20 \mathrm{GHz}$ and $5.80 \mathrm{GHz}$. The locations of these Uslots did not affect the notch frequencies apparently and were chosen to give the best impedance matching. The resulting four-band antenna had frequency ratios of $f_{2} / f_{1}=$ $1.09, f_{3} / f_{1}=1.52$ and $f_{4} / f_{1}=1.70$, where $f_{4}, f_{3}, f_{2}$ and $f_{1}$ were the center frequencies of the upper, middle and lower frequency bands. Because of the fabrication and assembly errors with the longest U-slot $\left(l_{7}+l_{8}+w_{4}-2 m_{4}\right)$, the discrepancy between theoretical and experimental results in the first, second and third frequency bands. This will be discussing further.

The measured and simulated radiation patterns in the E-plane and H-plane at $3.35 \mathrm{GHz}, 3.70 \mathrm{GHz}, 5.20 \mathrm{GHz}$ and $5.80 \mathrm{GHz}$ are shown in Figs. 5-8. It can be seen that all the crosspolarization levels are below $-10 \mathrm{~dB}$ in both E-plane and $\mathrm{H}$ plane. The measured and simulated peak gains of the proposed antenna are shown in Fig. 9. It can be seen that the measured peak gains of the antenna are 7.6 dBi, 8.6 dBi, 8.5 dBi and 9.0 dBi. All the gains of the notch bands are below $0 \mathrm{dBi}$. For simulation of ground plane size variation investigation of the effect of ground size on antenna gain was carried out. The peak gains of the four bands were only $1.62 \mathrm{dBi}, 3.48 \mathrm{dBi}, 7.51 \mathrm{dBi}$ and $8.37 \mathrm{dBi}$, when peak dimensions of ground and patch were same. When dimensions of the ground increases, gains show no significant change, when increase in four bands. This size was chosen for our Fig. 10 and it shows the photograph of the antenna.

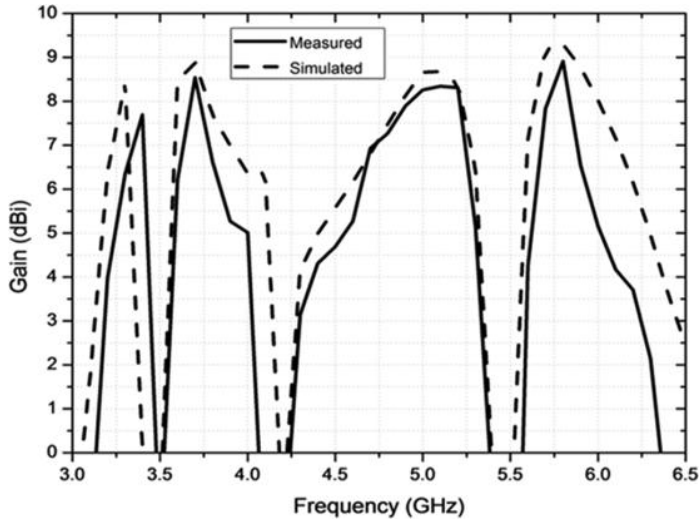

Fig 5: Measured and simulated gains of the antenna.

shows that the introduction of the fourth U-slot at about $3.5 \mathrm{GHz}$ in the patch resulted in a notch centered .The total length of the longest U-slot $\left(l_{7}+l_{8}+w_{4}-2 m_{4}\right)$ is 44.8 $\mathrm{mm}$, which is consistent with the analysis in Section II. Owing to the limited adjustable space of width $w_{3}$, an asymmetrical topology was employed to fine-tune the total length of the Uslot. In this design, was taken as the variable for the asymmetrical slot. By fine-tuning, desired frequency points can be obtained Fig 11. The fine-tuning of did not affect

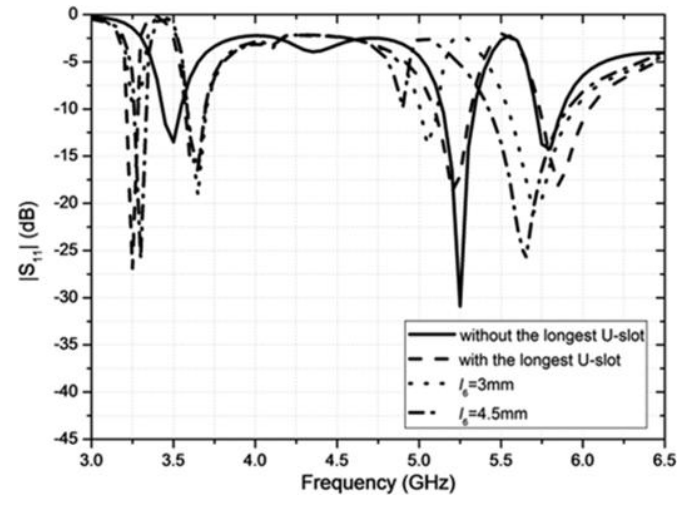

Fig 6: Effect of the longest U-slot and simulated reflection coefficients on different $l_{e}$.

The antenna which are fabricated prototype is shown in fig 2.By using low cost printed circuit board process (PCB) each parts of the antenna are fabricated by using it. For getting the remaining thickness of SIW substrate, two 20 mil and $32 \mathrm{mil}$ layers of Rogers 4003 are glued to each other. Overall dimension is $34 \times 69 \mathrm{~mm}^{2}$. SIW is fed by a $3 \mathrm{~mm}$ width microstrip line and it is matched with $50 \Omega$ impedance. substrate are etched with dimension $40 \times 56 \mathrm{~mm}^{2}$ and they are etched by radiating patched and radiating patched have dimension of $6.77 \times 10.8 \mathrm{~mm}^{2}$. By using four stands on the SIW substrate of patches is kept at a distance of $1 \mathrm{~mm}$. 


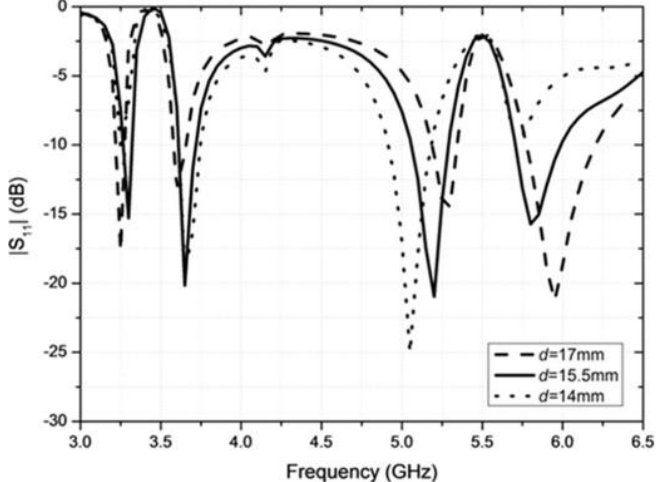

Fig 7: Simulated reflection coefficients for the $d$, the current distribution seriously and resulted only in an asymmetrical U-slot.

For keeping the FSS at a distance of $5 \mathrm{~mm}$, four Teflon made legs are used. And for reducing the cross polarization level this spacing is adjusted and at the same interval of time make the loading effect of the FSS be neglected. Measured return losses and the simulated of antenna are shown in fig 6. Its noticed that antenna has two resonances having wide $10 \mathrm{db}$ return loss bandwidth of $7.3 \%$ and $7.76 \%$ at 10 and $12 \mathrm{GHz}$.

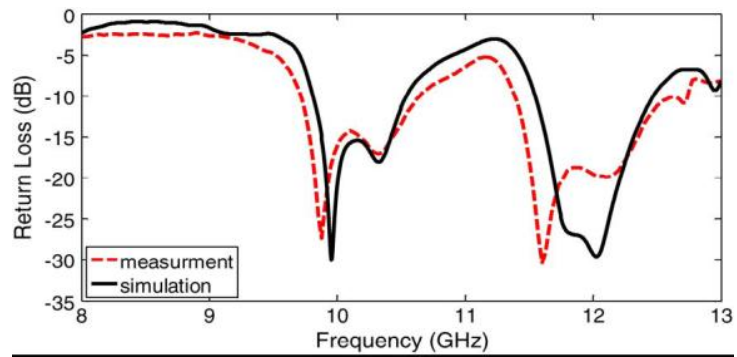

Fig 8: Return loss of the FSS-loaded antenna in X-band.

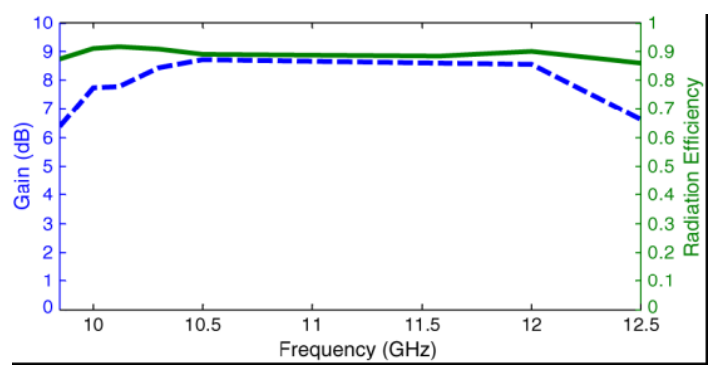

Fig 9: Simulated antenna gain

Higher bandwidth directed polarization and the low band is created by longitudinal slot .It has directed polarization and are provided by transverse slot with short length. Fig 7 shows the radiation pattern of antenna in a frequency close to centre frequency of each band. Good agreement between simulated and measured results has been observed .Its lower than $20 \mathrm{db}$ of good cross polarization level. And it is perpendicular to the antenna with maximum gain of $8.5 \mathrm{db}$, and then it is noticed at first and second bands. In two operation bands the simulated gain and radiation efficiency is shown in figure 8.As the radiating element, this antenna has higher gain of a single slot or a single patch as the radiating element. Efficiency of this antenna is close to $90 \%$ in its operation bands.

\section{CONCLUSION}

There is a simple geometry of a single-layer single patch four band four asymmetrical U slot patch antenna fed by a coaxial probe. $\mathrm{U}$ slot antenna is principally designed for the operating frequencies at $3.35 \mathrm{GHz}, 3.70 \mathrm{GHz}$, for $\mathrm{S}$ band and for $\mathrm{C}$ band it operates at $5.20 \mathrm{GHz}$ and $5.80 \mathrm{GHz}$ and they are the WiMax and WLAN bands. The (SIW) fed patch array antenna having two band with different polarization, the longitudinal and transverse slots are set on SIW for increasing the gain and bandwidth. This antenna provides the two frequency bands at $10 \mathrm{GHz}$ and $12 \mathrm{GHz}$. This provides the more directivity with the help of two longitudinal and transverse slots.

\section{REFERENCES}

[1] J. H. Lu, C. L. Tang, and K. L. Wong, July 2000, "Novel dual-frequency and board-band designs of slot-loaded equilateral triangular microstrip antennas," IEEE Trans,vol. 48, no. 7, pp. 1048-1054.

[2] S. Weigand, G. H. Pan, and J. T. Bernhard, Mar. 2003 , "Analysis and design of broad-band single-layer rectangular U-slot microstrip patch antennas," IEEE Trans. Antennas Propag., vol. 51, no. 3, pp. 457-468.

[3] P. Nayeri, K. F. Lee, A. Z. Elsherbeni, and F. Yang, Oct. 2011, "Dual-band circularly polarized antennas using stacked patches with asymmetric U-slots," IEEE Antennas Wireless Propag. Lett., vol. 10, pp. 492-495.

[4] Y. Cao, B. Yuan, and G.Wang, Sep. 2011, "A compact multiband open-ended slot antenna for mobile handsets," IEEE Antennas Wireless Propag. Lett., vol. 10, pp. 911914.

[5] B. Yildirim, Jun. 2011, "Multiband and compact WCDMA/WLAN antenna for mobile equipment," IEEE Antennas Wireless Propag. Lett, vol. 10, pp. 14-16.

[6] Shuo Liu, Shi-Shan Qi, Wen Wu, and Da-Gang Fang, Sep. 2014, "Single-Layer Single-Patch Four-Band Asymmetrical U-Slot Patch Antenna" IEEE Transactions On Antennas And Propagation, vol. 62, no. 9.

[7] L. Sabri, N. Amiri, and K. Forooraghi, , 2014, "DualBand and Dual-Polarized SIW-Fed Microstrip Patch Antenna" IEEE Antennas And Wireless Propagation Letters, vol. 13 .

[8] K. Gosalia and G. Lazzi, Sep. 2003, "Reduced size, dualpolarized microstrip patch antenna for wireless communications," IEEE Trans. Antennas Propag., vol. 51, no. 9, pp. 2182-2186.

[9] D. T. Notis, P. C. Liakou, and D. P. Chrissoulidis, Oct. 2004, "Dual polarized microstrip patch antenna, reduced in size by use of peripheral slits," in Proc. Eur. Conf. Wireless Technol., pp. 273-276.

[10] C.-Y.-D. Sim, C.-C. Chang, and J.-S. Row, Oct. 2009 , "Dual-feed dual-polarized patch antenna with low cross polarization and high isolation," IEEE Trans. Antennas Propag., vol. 57, no. 10, pp. 3321-3324.

[11] M. Veysi, M. Kamyab, and A. Jafargholi, Feb. 2011 "Single-feed dual-band duallinearly- polarized proximity-coupled patch antenna," IEEE Antennas Propag. Mag., vol. 53, no. 1, pp. 90-96.

[12] L. Yan andW. Hong, Feb. 2005 "Investigations on the propagation characteristics of the substrate integrated waveguide based on the method of lines," IEEE Proc., Microw., Antennas Propag., vol. 152, no. 1, pp. 35-42. 